\title{
Warum auch die Sprachenfrage die Zukunft unserer Demokratien bedroht.
}

\section{Eine Polemik}

\section{Vorbemerkung}

Harmloser darf man die Überschrift eines Artikels nicht formulieren, wenn man diesem überaus wichtigen Thema nicht wenigstens ein Mindestmaß an Aufmerksamkeit sichern möchte. Und dabei ist doch zumindest das Wichtigste zum Thema eigentlich schon sehr häufig gesagt worden.

\section{Einleitung}

Wir leben in einer Zeit, in der das Englische zur weltweit dominierenden Verkehrs- und Handelssprache geworden ist. Die Gründe hierfür sind vielfältig benannt worden. Es sind die Folgen des British Empire und der damit einhergehenden Kolonisation zahlreicher Länder dieser Welt, in denen die Sprache (vor allem auch als Amtssprache) geblieben ist. Und es ist die wirtschaftlich und militärisch dominante Rolle der USA seit dem Zweiten Weltkrieg. Englisch scheint omnipräsent zu sein, auch wenn man sich zumindest in vier Kontinenten dann doch immer wieder wundern muss, wie wenig weit man mit Englisch kommen kann, wenn man die ausgetretenen Pfade
Die bedeutende Rolle des Englischen als weltweite Wissenschaftssprache ist unbestritten. Vor einer stetig voranschreitenden Ausweitung der englischsprachigen Lehre an Hochschulen in nichtenglischsprachigen Ländern muss jedoch ausdrücklich gewarnt werden. Und das gilt erst recht, wenn die Lehre auch noch ersatzlos auf Studiengänge im BA-Studium ausgeweitet werden soll. Und da muss auch Polemik erlaubt sein. 
des Tourismus oder auch höhere Kommunikationsebenen der Wirtschaft oder der Politik verlässt; wobei es in der Politik weltweit recht häufig auch nur auf der zweiten oder gar dritten Ebene ausreichend Personal gibt, die des Englischen auf einer höheren Kompetenzstufe mächtig sind. Anders sieht es vordergründig tatsächlich aus, wenn man sich die Situation in der weltweiten Wissenschaft vergegenwärtigt, in der Englisch dominierend ist und immer wieder als Lingua franca ausgerufen wurde und wird, auch wenn bereits häufig darauf verwiesen wurde, wie wenig der Vergleich mit der Rolle des Lateins im Mittelalter haltbar ist. ${ }^{1}$ Deutlicher wird, hinsichtlich der Funktionalität des Englischen, Winfried Thielmann in einer Fußnote:

Mit Blick auf die Diskussion um mögliche lingua-franca-Nutzungen des Englischen im Bereich der Wissenschaft möchte ich mir die Bemerkung gestatten, dass solche hochkomplexen sprachlichen Bearbeitungen der Epistemizität wissenschaftlichen Wissens nur in einer voll ausgebauten und auch auf diesem Niveau beherrschten wissenschaftlichen Varietät, und eben nicht in einer lingua franca, erfolgen können. ${ }^{2}$

Die bereits offensichtlichen und die absehbaren Folgen des seit längerem voranschreitenden Prozesses der Anglifizierung der Wissenschaftssprache lassen sich auf verschiedenen Ebenen verorten. Hier soll wenigstens kurz auf drei Ebenen etwas näher eingegangen werden: Erstens der Bedeutungsverlust des Deutschen als Wissenschaftssprache mit weitreichenden Konsequenzen, zweitens die Umgestaltung der deutschen Hochschullehre mit seinen unbestreitbaren Vor- und vor allem auch den gegebenen Nachteilen sowie drittens die Folgen für zahlreiche Gesellschaften weltweit, in denen nicht Englisch die Muttersprache ist.

\section{Deutsch als Wissenschaftssprache und die Dominanz des Englischen}

Der Philosoph und Kritiker unserer Bildungsentwicklung Konrad Liessmann hat einschränkend formuliert:

Man muß die rasche Etablierung des Englischen als alleinige Wissenschaftssprache ja nicht gleich als puren Sprachimperialismus diskreditieren - bei allem Wettbewerbsvorteil, den native speakers gegenüber jenen haben, die diese Sprache erst erwerben müssen, sind die Erleichterungen in der Kommunikation unübersehbar -, aber man soll auch nicht die Augen davor verschließen, daß in dem Maße, in dem nationale Sprachen aufhören, auch Wissenschaftssprachen zu sein, genau jenes Motiv außer Kraft gesetzt wird, das in und durch die Aufklärung dazu geführt hatte, die einstige Wissenschaftssprache Latein durch die Volkssprachen zu ersetzen. ${ }^{3}$

1 Vgl. z.B. Trabant: Über die Lingua franca der Wissenschaft.

2 Thielmann: Illokutionsstrukturen wissenschaftlicher Texte, S. 17.

3 Liessmann: Theorie der Unbildung, S. 132. 
Man kann die Entwicklung zum Englischen als dominierende Wissenschaftssprache der Welt aber durchaus unter sprachimperialistischen Gesichtspunkten betrachten. Konrad Ehlich stellte bereits 2000 fest, dass »[s]elbstverständlich [...] die deutlich kulturimperialistischen Züge des Anglifizierungsprozesses der Wissenschaftskommunikation nicht verborgen geblieben [seien] «. ${ }^{4}$ Siegfried Gehrmann wird hier sehr viel deutlicher, wenn er bereits in der Unterüberschrift eines Artikels die "Sprachlichkeit von Wissenschaft als Teil einer neuen Weltordnung « bezeichnet. ${ }^{5}$ Mit Bezug auf einen Artikel von Herfried Münkler in »Die Zeit« (2014), in dem dieser verdeutlicht, dass es weltpolitisch gesehen im 21 . Jahrhundert nicht mehr notwendigerweise »um die Inbesitznahme eines strategisch wichtigen Stücks Boden [...] [gehe], sondern um die Kontrolle und Steuerung eines Gesamtzusammenhangs «, der auf die »Kontrolle von Strömen angelegt [sei]: Strömen von Kapital und Informationen, Gütern und Dienstleistungen, Rohstoffen und Personen «. ${ }^{6}$ Und sprachenpolitisch gehe es bei der Durchsetzung einer derartigen neuen Weltordnung

immer auch um die Kontrolle und Steuerung von Kommunikationsvorgängen, mit dem Ziel, die Welt sprachlich und kommunikativ so zu entterritorialisieren, so dass Informationsströme unabhängig von nationalsprachlich differenten Weltdeutungen gelenkt und beherrscht werden können. ${ }^{7}$

\section{Und dies setze}

neben der Existenz einer sich imperial durchsetzenden Weltmacht auch das Vorhandensein und die Wirkmächtigkeit einer Welteinheitssprache voraus, die imstande ist, die Welt im Sinne imperialer Logik einer einheitlichen und universell gültigen Rekategorisierung zu unterwerfen und alternative sprachliche Kategorisierungs- und Weltdeutungsmuster aufzuheben oder zumindest in zentralen Bereichen gesellschaftlicher Lenkung und Machtausübung abzuschwächen oder diskursiv zu marginalisieren. ${ }^{8}$

Es lässt sich wohl kaum klarer formulieren. Wir haben nicht nur zu konstatieren, dass "gewisse Amerikanisierungstendenzen in der emergierenden Globalkultur [...] wie die Macht der amerikanischen Filmindustrie oder die Rolle der englischen Sprache « ${ }^{9}$ einen weltweiten Einfluss auf alle Kulturen haben, dass global agierende Megakonzerne wie Amazon, Facebook, Google oder Microsoft unser gesamtes Leben weltweit folgenreich bestimmen und dabei auch noch die Macht haben, sich (bislang) weitgehend über nationale

Ehlich: Deutsch als Wissenschaftssprache für das 21. Jahrhundert, S. 57.

5 Gehrmann: Die Kontrolle des Fluiden.

6 Münkler: Soldat ohne Staat. Vgl. Gehrmann: Die Kontrolle des Fluiden, S. 117.

7 Gehrmann: Die Kontrolle des Fluiden, S. 118.

8 Ebd.

9 Altmayer: Landeskunde im Globalisierungskontext, S. 8. 
Steuergesetzgebungen hinwegsetzen zu können. Wir müssen zudem feststellen, dass weltweit alle Volkswirtschaften von der Einschätzung dreier US-amerikanischer Rating Agenturen, nämlich Standard \& Poors, Moody's und Fitch, abhängig sind. Die Forderung nach der Schaffung einer möglichst unabhängigen (konkurrierenden) europäischen Rating Agentur verlief bisher erfolglos, auch wenn Versuche unternommen wurden. Weltweit sind Volkswirtschaften und damit Nationen weiterhin von Bewertungen dieser drei Agenturen in hohem Maße abhängig. In gewisser Weise ähnlich erfolglos verlief bisher die Forderung nach einer europäischen Einrichtung, die der Dominanz des US-amerikanischen Citation-Index und dem die Wissenschaft maßgeblich verändernden Journal Impact Factor etwas entgegenzusetzen hätte. Die Unausgewogenheit und die Folgen der aktuellen Praxis für die weltweite Wissenschaft hat Gehrmann deutlich dargestellt. ${ }^{10}$ Es gibt sogar vermehrt ernstzunehmende Hinweise, dass renommierte englischsprachige Zeitschriften nahelegen, keine nicht-englischsprachige Sekundärliteratur zu zitieren. Und welche weiteren obskuren Folgen die anglo-amerikanische Wissenschafts- und Publikationspraxis haben kann, sei an einem eigenen Beispiel verdeutlicht: Meine - und dieses markante Beispiel möge hier erlaubt sein - gerade noch neueste Publikation hat folgenden Titel: Informed consent at stake? Language barriers in medical interactions with immigrant anaesthetists. A conversation analytical study. ${ }^{11}$ Wie kommt ein DaF-ler zu einer derartigen Publikation in einer englischsprachigen medizinischen Zeitschrift (»BMC Health Services Research«)? Diese Tatsache erklärt sich aus meiner Betreuung einer Dissertation, die die Schwierigkeiten der Kommunikation der in einem deutschen Krankenhaus arbeitenden nicht-muttersprachlichen Ärztinnen und Ärzte mit einheimischen Patientinnen und Patienten in medizinischen Aufklärungsgesprächen eingehend beleuchtet und den daraus abzuleitenden sprachlichen Schulungsbedarf herausarbeitet. ${ }^{12}$ Der Chefarzt des für die Untersuchung maßgeblichen Bielefelder Krankenhauses, Prof. Dr. Rahe-Meyer, der die Erhebung der Originaldaten überhaupt erst ermöglicht hat (Mitautor), hat sicherlich völlig zu Recht darauf gedrungen, dass Damaris Borowski ihre empirisch gewonnenen Erkenntnisse auch der medizinischen Fachwelt zur Kenntnis gibt. Daraus resultierte allerdings folgendes: Um überhaupt eine Chance auf Annahme des Artikels durch eine renommierte medizinische Zeitschrift mit Peer-review-Verfahren zu haben, mussten die drei DaF- 
ler*innen (mit Professorenstatus), die abschließend für die erfolgreiche Disputation der Promotion zuständig waren, mitgenannt werden. Und der die Arbeit begleitende Chefarzt musste an letzter Stelle angeführt werden. Sonst, so die überzeugend vorgetragene Argumentation, hätte es keine Chance auf eine Veröffentlichung gegeben. Es wird nachvollziehbar sein, dass wir aus dem Bereich DaF einer derartigen (auch für unsere Doktorandin potentiell wichtigen) Veröffentlichung nicht im Weg stehen wollten, auch wenn wir allenfalls kleine Korrekturvorschläge einbringen konnten. Sehr erstaunt hat uns dann allerdings nach einer auch uns erfreuenden Annahme des Artikels, dass die dafür anfallenden, absolut nicht unerheblichen Publikationskosten von dem Krankenhaus übernommen wurden, an dem die Forschung stattgefunden hat. Was können wir für die Zukunft unseres Fachs daraus ableiten? Möglicherweise nichts Gutes.

Noch können und dürfen wir im Fach Deutsch als Fremdsprache und in der Germanistik (aber das betrifft wohl auch nur noch den Zweig der Literaturwissenschaft und nicht mehr den der Linguistik) in deutscher Sprache veröffentlichen, ohne einen weiteren Schaden außer der über das Fach hinausgehenden Ignoranz hinnehmen zu müssen. Man muss es dann wohl einfach ertragen können, wenn - wie bereits vorgekommen - Erkenntnisse in der internationalen Forschung zur >Second-language acquisition< als neu ausgegeben werden, auch wenn diese bereits seit langer Zeit in der deutschsprachigen Zweitsprachenerwerbsforschung bekannt waren. Das ist einfach eine Folge von `English only . Auch wichtige Werke von Jürgen Habermas sind erst sehr spät ins Englische übersetzt worden und damit dem größten Teil der internationalen Wissenschaftlergemeinschaft erst mit Verzögerung zugänglich geworden. Wie wird sich eigentlich die internationale Geschichtswissenschaft weiterentwickeln, wenn in einem jetzt schon absehbaren Maße zukünftige Wissenschaftlergenerationen außerhalb des deutschsprachigen Raums (und das gilt natürlich auch für andere Sprachen) Quellentexte nur noch auf Englisch lesen und verstehen können? Wie soll dann zukünftig internationale Geschichtsschreibung aussehen und wie fundiert kann sie überhaupt noch sein?

\section{Die Lehrsprache an deutschen Hochschulen}

Fast schien es schon so, als ob die Einrichtung englischsprachiger Studiengänge an deutschen Hochschulen den Höhepunkt der Realisierungen sowie der damit verbundenen Marketingmaßnahmen im Rahmen der Internationalisierungsbemühungen bereits überschritten hätte, als dann vor kur- 
zem für das Bundesland Bayern die Nachricht auftauchte, dass »Bayerns Ministerpräsident Markus Söder (CSU) [...] verkündet [habe], dass der technologische Bereich der Hochschulen vollkommen internationalisiert werden soll und technische Studiengänge durchweg englischsprachig sein sollen ${ }^{13}{ }^{13}$ Dafür soll eine Gesetzesänderung die Möglichkeit schaffen, fremdsprachige Studiengänge auch im grundständigen Bereich einzuführen. Die weitere Berichterstattung im gleichen Artikel der »FAZ« offenbart bereits die Probleme. Die Realisierung der Pläne scheint zwar auf politischer Ebene möglich.

Wenn da nur nicht das Problem mit den Englischkenntnissen der Studenten wäre. Denn Fremdsprachenkenntnisse können nicht von den Studenten verlangt werden. »Insoweit muss bis zur Gesetzesänderung auf die Selbsteinschätzung der Studienbewerberinnen und -bewerber vertraut werden.« Englischsprachige Studiengänge könnten also auch von Bewerbern gewählt werden, deren Englisch das eigentlich für ein Studium nötige C2-Niveau nach Europäischem Referenzrahmen bei weitem nicht erreicht. ${ }^{14}$

Man wird in sehr vielen Fällen froh sein können, wenn tatsächlich die das Studium neu beginnenden deutschen Studierenden in den geplanten Studiengängen - sogar selbst mit einem bayrischen Abitur - über das Niveau B2/C1 verfügen, welches nach TestDaF und der attestierten Niveaustufe 4 in allen vier Fertigkeitsbereichen dem vorgeschriebenen Eingangssprachniveau in der Lehrsprache Deutsch für internationale Studierende an deutschen Hochschulen entspricht, wenn die einzelne Hochschule nicht für sich ein anderes Zugangsniveau (und wenn auch nur für einzelne Fächer) festgelegt hat. Dabei muss festgehalten werden, dass mit dem von TestDaF auf $>4 \mathrm{x} 4$ < attestierten Niveau (B2/C1) nicht die generelle, umfassende Studierfähigkeit bescheinigt werden kann, sondern eine sprachliche Ausgangslage erfasst wird, bei der man ursprünglich davon ausging, dass das Leben in einer deutschsprachigen Umwelt, die intensiven Wissenschaftskontakte im Hochschulalltag und die Lektüre deutschsprachiger wissenschaftlicher Texte dazu führen werden, dass das erforderliche höhere sprachliche Niveau quasi nebenbei erreicht werden kann. Dies war ein Fehlschluss, wie inzwischen mehrfach festgestellt wurde. Dazu beispielsweise Yu Chen:

Den wohl jüngsten Bericht dazu findet man in ZEIT in der Ausgabe vom 23.03.2017: unverständliche Hausarbeiten auf Deutsch, die offensichtlich mithilfe eines GoogleÜbersetzers erstellt wurden; unverständliche Vorträge, egal auf Deutsch oder Englisch. Konkrete Erfahrungen von Lehrpersonen bestätigen immer wieder, dass mangelnde 
Deutschkenntnisse ein großes Problem des Ausländerstudiums darstellen (Oswalt \& Adams 2017). ${ }^{15}$

Warum sollte das in englischsprachigen Studiengängen anders sein, wenn sogar das alltägliche englischsprachige Umfeld fehlt? Da hilft vermutlich sogar der zunehmende Konsum englischsprachiger Filmserien (z.B. per Netflix), der tatsächlich die Englischkompetenz deutscher Studierender zu fördern scheint, nur bedingt, weil in Spielfilmserien Fach- und Wissenschaftssprache nur sehr begrenzt eine Rolle spielt. Selbst in der Tagespresse weiß man im bereits oben erwähnten Artikel inzwischen durchaus schon mitzuteilen, warum die Einführung englischsprachiger Studiengänge entgegen dem damit verbreiteten Flair von Internationalisierung und Weltläufigkeit letztlich nicht unproblematisch ist:

Aus anderen europäischen Ländern, selbst skandinavischen, denen bessere Englischkenntnisse nachgesagt werden, ist bekannt, dass Studenten naturwissenschaftlichen Vorlesungen, die auf Englisch gehalten wurden, weit schlechter folgen konnten. Untersuchungen aus Schweden und den Niederlanden zeigen Ähnliches: Danach hat der universitäre Unterricht in Englisch das Leistungsniveau erheblich gesenkt. Selbst der dänische Sprachrat stellte fest, dass das fachliche Niveau an Universitäten sinkt, wenn fast vollständig auf Englisch gelehrt wird. ${ }^{16}$

Derartige Sachargumente zählen jedoch offensichtlich nicht; dann schon eher die Hoffnung, dass sich durch die geplante bayrische Maßnahme akademisches Spitzenpersonal aus dem Brexit-geplagten Großbritannien wird anlocken lassen.

Aktuell (Dezember 2019) weist der Hochschulkompass der Hochschulrektorenkompetenz 1531 englischsprachige Studiengänge an den deutschen Hochschulen aus. In einer DAAD-Studie heißt es dazu:

Durch die wachsende Globalisierung, die demografische Entwicklung und die steigende Nachfrage des Arbeitsmarktes nach Absolventen mit internationalen Kompetenzen sind die deutschen Hochschulen immer starker gefordert, sich bei der Rekrutierung und Ausbildung von Studierenden im weltweiten Bildungsmarkt zu behaupten. Die Einrichtung englischsprachiger Studiengänge ist eine Antwort auf diese Herausforderungen. Entsprechende Studienangebote sind für international mobile Studierende attraktiv, da Sprachbarrieren entfallen bzw. gemildert werden, und schaffen auch für die deutschen Teilnehmerinnen und Teilnehmer neue Chancen zum Erwerb interkultureller und sprachlicher Kompetenzen. Seit dem Beginn des Bologna-Prozesses gegen Ende der 1990er Jahre hat sich die Zahl der englischsprachigen Studiengänge vervielfacht. In einer ersten Bestandsaufnahme, die von der Academic Cooperation Association (ACA) in Zusammenarbeit mit der Gesellschaft für Empirische Studien (GES) im Jahre 2001 durchgeführt wurde, konnten in Deutschland lediglich 180 entsprechende Studienangebote identifiziert werden. Bis zum Jahr 2007 hat sich diese Zahl nur geringfügig auf 214 erhöht. 
Einen starken Zuwachs hat es erst in den letzten Jahren gegeben. Im Juni 2017 waren im HRK-Hochschulkompass 1.210 englischsprachige Studiengange registriert; die Zahl hat sich also innerhalb nur weniger Jahre versechsfacht. ${ }^{17}$

Problematisch angesichts dieser wie eine Erfolgsmitteilung klingenden Information bleibt natürlich die Frage nach dem Erfolg und dem damit nicht unerheblich zusammenhängenden Faktor Sprache. Die in der Studie von Fandrych und Sedlaczek ${ }^{18}$ nachgewiesenen, z.T. eklatanten sprachlichen Mängel auf Seiten der Studierenden wie vor allem auch Lehrenden in den englischsprachigen Studiengängen an deutschen Hochschulen dürften inzwischen nicht einfach völlig verschwunden sein. Die Hochschulrektorenkonferenz hat bereits vor Jahren auf dieses Dilemma hingewiesen: »Allerdings verfügen nicht alle Lehrenden zwangsläufig über die erforderlichen Kenntnisse, um auch auf Englisch exzellente Lehre sicher gewährleisten zu können. ${ }^{19}$ Und es dürfte auch weiterhin gelten, dass »eine große Zahl deutscher HochschullehrerInnen sich hinsichtlich ihrer tatsächlichen Englischsprachkompetenz (manchmal sogar sehr) überschätzt «. ${ }^{20}$ Insgesamt kann Folgendes als gesichert gelten:

Wenn weder Studierende noch Lehrende, Gesellschaft oder Lernumgebung anglophon sind, hat Lehre in englischer Sprache nachteilige Auswirkungen nicht nur auf die Qualität der Lehre und die Lernstoffrezeption, sondern auch auf die Institution Hochschule und letztlich auf die Gesellschaft und deren Integrationsfähigkeit insgesamt. ${ }^{21}$

\section{Wissenschaftssprache und Demokratie}

Unter der Überschrift Vertrauensverlust. Wie die Wissenschaft sich selbst schadet hat Jürgen Zöllner, langjähriger Bildungs- und Wissenschaftsminister sowie Senator für Bildung und Wissenschaft (von 1991 bis 2006 in Rheinland-Pfalz und von 2006 bis 2011 in Berlin), eine zentrale Aufgabe von Wissenschaft folgendermaßen beschrieben:

Wissenschaft hat die Pflicht, sich der Gesellschaft zu erklären und zwar in einer Sprache, die die Menschen verstehen. Ich meine hier ausdrücklich auch die deutsche Sprache. Wer, wenn nicht die Kultur- und Gesellschaftswissenschaften, soll heute der Gesellschaft das Orientierungswissen vermitteln, dass sie in einer globalisierten Welt mit täglich einschneidenden Veränderungen mehr braucht denn je. Die Wissenschaft wird aber nur

17 Internationalität an deutschen Hochschulen, S. 100.

18 Fandrych/Sedlaczek: »I need German in my life...".

19 Sprachenpolitik an deutschen Hochschulen.

20 Koreik: Die Sprachenfrage in internationalen Studiengängen, S. 102.

21 Rösch/Tolkiehn/Lehnert: Die Landessprache in der Lehre, S. 13. 
dann die breite Bevölkerung und Politik erreichen, wenn es auch auf Deutsch geschieht. Ganz abgesehen von der kulturellen Dimension, dass die deutsche Sprache verarmt, wenn in ihr wissenschaftliche Begriffe gar nicht mehr herangebildet werden. ${ }^{22}$

Damit werden wesentliche Aspekte sehr klar benannt!

Es ist für die damit befasste Wissenschaftlergemeinschaft sehr offensichtlich, welche Folgen eine zunehmende Eingrenzung der Wissenschaft und vor allem auch der zukünftigen Forschungserträge auf die dominierende Wissenschaftssprache Englisch hat. Das Potential des Denkens und damit zwangsläufig auch Handelns wird eingeschränkt werden. Der Verlust der Artenvielfalt weltweit ist als Vergleich naheliegend. Biologinnen und Biologen warnen vor unumkehrbaren Folgen. Man kann das als sentimentale Panikmache abtun. Sprachen in der Welt sterben eben aus. Warum auch nicht? Schließlich hat angesichts möglicher anderer Optionen in der Entwicklung der Menschheit auch nur der Homo sapiens überlebt. Da scheint es doch nur logisch zu sein, wenn wir endlich das Dilemma des Turmbaus zu Babel überwinden und zumindest in der Wissenschaft in einer Sprache kommunizieren (können). Das Problem ist nur, dass damit offensichtlich Erkenntnisverluste in Kauf genommen werden. Und das gilt nachweisbar nicht nur für die geistes- und sozialwissenschaftliche Sphäre, sondern auch für die naturwissenschaftlich-technische Welt. Aber das kümmert in der weltweiten Bildungsplanung kaum jemanden. Wichtig sind ja nur noch quantifizierbare Ergebnisse. Und dazu gehört der prozentual nachweisbare Grad der Internationalisierung, wobei meist geflissentlich übersehen wird, dass dieser in anglo-amerikanischen Ländern eben gerade auch ein Indikator für die nicht unerheblichen Einnahmen der Hochschulen durch die Studiengebühren internationaler Studierender geworden ist. Die Einnahmen durch Studiengebühren an Hochschulen oder auch durch Sprachkurse und die darauf basierenden Sprachtests für den Hochschulzugang können mittlerweile volkswirtschaftlich relevante Dimensionen annehmen. Und um der zahlenden Klientel zu genügen, müssen dann nicht selten auch bisher gültige akademische Standards gesenkt werden, damit angesichts der erhebliche Finanzen einbringenden Kundschaft auch in Form eines anerkannten Abschlusszeugnisses geliefert werden kann. Es ist nicht nur eine Noteninflation die Folge, sondern zugleich eine fast überall stattfindende Absenkung des bisher üblichen erforderten Leistungsniveaus.

Natürlich kann man weiterhin behaupten, dass weltweit an den Hochschulen der verschiedensten Länder angebotene Studiengänge auf Englisch der Internationalisierung dienen. Ja, das tun sie! Aber um welchen Preis? 
Eine Nivellierung des inhaltlichen Niveaus ist zumeist vonnöten, damit letztendlich Abschlusszertifikate ausgestellt werden können. Die Investitionen der Studierenden (bzw. die ihrer Eltern) müssen sich ja lohnen und die zukünftigen Einnahmen der die Zeugnisse ausgebenden Hochschule weiterhin gesichert bleiben.

Ein Studium in einer Fremdsprache führt bei einer nicht wirklich ausreichenden Sprachkompetenz zu Defiziten in der Erkenntnisaufnahme und -verarbeitung. Erfolgt die Vermittlung der Studieninhalte dann auch noch von einer Lehrperson, die selbst die Lehrsprache nicht auf muttersprachlichem Niveau beherrscht, sind die potentiellen Erkenntnisgewinne möglicherweise gravierend. Studien wie die von Airy und Lindner ${ }^{23}$ deuten darauf hin. Und dabei geht es meistens offensichtlich nicht um eine adäquate Fachlexik oder auch allgemein um Fachsprache, sondern eher um Defizite im allgemeinsprachlichen, allenfalls bildungssprachlichen Bereich. Hierfür sind die eindrücklichen Beispiele bei Thielmann zu einer »Vorlesung im lingua-franca-Englisch im Fach Chemie « bezeichnend. ${ }^{24}$ Und das gilt selbstverständlich nicht nur für die englische Sprache an deutschen oder deutschsprachigen Hochschulen, sondern auch für die Vermittlungssprache Deutsch an ausländischen Hochschulen. ${ }^{25}$ Sprachkompetenz bleibt ein absolut entscheidendes Kriterium für die Qualität von Wissenschaft und Forschung. Und natürlich kann man behaupten, dass es nicht auf die absolute Perfektion der Sprachbeherrschung ankomme, um relevante Inhalte zu kommunizieren. Und das ist ja auch richtig. Und natürlich kann man auch Autos produzieren, die nur durch gezielte Manipulationen den vorgegebenen Normen entsprechen. Und selbstverständlich kann man auch behaupten, dass die Sprachenfrage überschätzt werde und die weltweit erhobenen Zahlen ja zeigen würden, wie gut die Internationalisierung an Hochschulen angesichts der nicht nur in Deutschland gestiegenen Quoten funktioniert, wie man eben auch behaupten kann, dass es keinen Klimawandel gibt.

Wenn die deutsche Sprache jedoch nicht mehr Wissenschaftssprache ist und unsere auch auf bestimmte Fachgebiete spezialisierten Journalisten immer stärker in die Verlegenheit geraten, wichtige Erkenntnisse z.B. in der Gentechnik oder der Atomphysik in englischen Begriffen erfassen zu müssen, da es keine deutschen mehr dafür gibt, bekommen wir ein Legitimationsproblem, weil damit gleichzeitig klar ist, dass große Teile der Bevölkerung nicht mehr werden nachvollziehen können, worum es geht 
und was die grundlegenden Entscheidungskriterien sind oder sein könnten. Man mag dagegen einwenden, dass ein Großteil der Bevölkerung sowieso nie richtig verstanden hat, worum es letztlich bei zukunftsweisenden Forschungserträgen wirklich geht und ging. Aber sprachlich wäre es zumindest möglich gewesen. Wenn jedoch neue wichtige wissenschaftliche Erkenntnisse partiell nur noch auf Englisch kommuniziert werden können, weil es nicht einmal mehr deutsche Begriffe gibt, wird dies das Gefühl verstärken, dass die da oben sowieso machen, was sie wollen. Und zu welchen Ergebnissen das führen kann, haben wir bei den letzten Wahlen insbesondere im Osten Deutschlands erlebt. Wissenschaft muss transparent bleiben und verstanden werden können. Das impliziert auch eine Bringschuld der Wissenschaftlerinnen und Wissenschaftler. Sollten Sprachschwierigkeiten mangels einer weiter ausgebauten Wissenschaftsterminologie zu immer größeren Verständnisproblemen führen, scheint eine noch größere Abwendung von denen >da oben « unausweichlich zu sein. Wer will schon mit vielleicht nicht oder nicht sofort verständlichen englischsprachigen Begriffen konfrontiert werden? Und Populisten werden genau diese Unsicherheit des Verständnisses zu nutzen wissen. Somit wird die sprachliche Verständlichkeit auch komplizierter neuer wissenschaftlicher Erkenntnisse auch weiterhin ein entscheidender Bestandteil funktionierender Demokratien sein. Für den Erhalt der Sprachenvielfalt unserer Welt werden freitags keinesfalls Massen auf die Straßen gehen. Aber die Sprachübersetzungsprogramme (wie Google Translate oder DeepL) werden ja auch immer besser. Und allein schon die Tatsache, dass immer weniger Fachlexik wirklich übersetzt werden muss, wird sie auch noch schneller machen. Eine Maximierung des Outputs scheint ja schließlich das Entscheidende zu sein.

\section{Literaturverzeichnis}

Airy, John; Linder, Cadric: Language and the experience of learning university physics in Sweden. »European Journal of Physics« 27 (2006), S. 553-560.

Althaus, Hans-Joachim; Koreik, Uwe: Transnationale Bildung: Deutsch in mehrsprachigen Kontexten an Hochschulen. In: Förderung der deutschen Sprache weltweit. Vorschläge, Ansätze und Konzepte. Hgg. U. Ammon, G. Schmidt. Berlin, Boston: De Gruyter 2019, S. 215-240.

Altmayer, Claus: Landeskunde im Globalisierungskontext. Wozu noch Kultur im DaFUnterricht? In: Kulturelles Lernen im DaF/DaZ-Unterricht. Paradigmenwechsel in der Landeskunde. Hgg. Peter Haase, Michaela Höller. Göttingen: Universitätsverlag 2017, S. 3-22. 
Borowski, Damaris: Sprachliche Herausforderungen ausländischer Anästhesisten/innen bei Aufklärungsgesprächen. Eine gesprächsanalytische Studie zu Deutsch als Zweitsprache im Beruf. Berlin: Frank \& Timme 2018.

Borowski, Damaris; Koreik, Uwe; Ohm, Udo; Riemer, Claudia; Rahe-Meyer, Nils: Informed consent at stake? Language barriers in medical interactions with immigrant anaesthetists. A conversation analytical study. »BMC Health Services Research«19, 597 (2019).

Chen, Yu: Deutschkenntnisse ausländischer Studierender in Deutschland: Sprachstand und Fördermaßnahmen. In: Förderung der deutschen Sprache weltweit. Vorschläge, Ansätze und Konzepte. Hgg. U. Ammon, G. Schmidt. Berlin, Boston: De Gruyter 2019, S. 317-335.

Ehlich, Konrad: Deutsch als Wissenschaftssprache für das 21. Jahrhundert. »GfL« 1 (2000), S. 47-63.

Fandrych, Christian; Sedlaczek, Betina: "I need German in my life... "Eine empirische Studie zur Sprachsituation in englischsprachigen Studiengängen in Deutschland. Unter Mitarbeit von Erwin Tschirner und Beate Reinhold. Tübingen: Stauffenburg 2012.

Gehrmann, Siegfried: Die Kontrolle des Fluiden. Die Sprachlichkeit von Wissenschaft als Teil einer neuen Weltordnung. In: Bildungskonzepte und Lehrerbildung in europäischer Perspektive. Hgg. S. Gehrmann, J. Helmchen, M. Krüger-Potratz, F. Ragutt. Münster, New York: Waxmann 2015, S. 117-156.

Internationalität an deutschen Hochschulen. Erhebung von Profildaten 2017. Hgg. DAAD, AvH, HRK. Bonn: DAAD 2018 (=DAAD Studien). <http://docplayer.org/116093639Internationalitaet-an-deutschen-hochschulen.html> (Zugriff: 31.12.2019).

Koreik, Uwe: Die Sprachenfrage in internationalen Studiengängen. In: Internationale Studiengänge in den Geistes- und Kulturwissenschaften: Chancen, Perspektiven, Herausforderungen. Hg. Stephan Jolie. Bielefeld: UniversitätsVerlagWebler 2018, S. 95-107.

Liessmann, Konrad Paul: Theorie der Unbildung. Die Irrtümer der Wissensgesellschaft. Wien: Paul Zsolnay Verlag 2006.

Mocikat, Ralf: Die Diktatur der Zitatenindizes: Folgen für die Wissenskultur. In: „GAiA. Ökologische Perspektiven für Wissenschaft und Gesellschaft. Ecological Perspectives for Science and Society« 18/2 (2009), S. 100-103. Abrufbar unter: <http://adawis. de/fileadmin/user_upload/Seiten/Stellungnahmen/Publikationen_des_ADAWIS/ GAIA.pdf $>$.

Münkler, Herfried: Soldat ohne Staat. »Die Zeit« 39 (2014), 18.9.2014; »Zeit Online» 23.9.2014. <https://www.zeit.de/2014/39/weltpolitik-krieg-ukraine-islamischer-staat/ komplettansicht> (Zugriff: 31.12.2019).

Rösch, Olga; Tolkiehn, Günter; Lehnert, Ralf: Die Landessprache in der Lehre - welche Bedeutung kommt ihr bei der Internationalisierung zu? Internationalisierung und Landessprachen - Hochschullehre braucht beides! »Die Neue Hochschule« 6 (2019), S. 12-15.

Schmoll, Heike: Mehr rein englischsprachige Studiengänge. „Frankfurter Allgemeine Zeitung « 2014, aktualisiert am 14.10.2019. Abrufbar unter: <https://www.faz.net/ aktuell/karriere-hochschule/hochschulen-in-bayern-wollen-mehr-englischsprachigestudiengaenge-16431456.html> (letzter Zugriff: 5.12.2019).

Sprachenpolitik an deutschen Hochschulen. Empfehlung der 11. Mitgliederversammlung der Hochschulrektorenkonferenz am 22.11.2011. <https://www.hrk.de/fileadmin/_ migrated/content_uploads/Empfehlung_Sprachenpolitik_MV_22112011.pdf> (Zugriff: 9.12.2019)

Thielmann, Winfried: Illokutionsstrukturen wissenschaftlicher Texte im Hinblick auf den Umgang mit Wissen. »Zielsprache Deutsch« 42 (2015) 1, S. 3-20. 
Thielmann, Winfried: Sprachliche Voraussetzungen einer europäischen Wissensbildung (im Druck).

Trabant, Jürgen: Über die Lingua franca der Wissenschaft. In: Deutsch in der Wissenschaft. Ein politischer und wissenschaftlicher Diskurs. Hgg. H. Oberreuther, W. Krull, H. J. Meyer, K. Ehlich. München: Olzog 2012, S. 101-107.

Zöllner, Jürgen: Vertrauensverlust. Wie die Wissenschaft sich selbst schadet. »Der Tagesspiegel«13.1.2019. Abrufbar unter: <https://www.tages spiegel.de/wissen/vertrauensverlust-wie-die-wissenschaft-sich-selbstschadet/23836930.html> (Zugriff: 4.12.2019). 